\title{
Geography should be taught at medical school
}



Geography in the South African (SA) context is as singular a driver of health-seeking behaviour as the burden of disease. This is the dilemma facing the rural patient presented with the 'choice' of health facilities where access could mean long transit times, or as is often the case, an expensive commute..$^{[1]}$

Geography, and more specifically infrastructure (or the lack thereof in rural areas), has long been identified as affecting access to health services, and by extension, disease outcome. ${ }^{[1,2]}$ Nowhere is this more acutely felt than in the field of emergency medicine, where notions such as definitive care and the 'golden hour' seem to form an integral part of the therapeutic discourse. While health system 'engineers' and decision-makers appear to be cognisant of this challenge, it seldom manifests into equitable, affordable, available, acceptable and accommodating access. This manifests itself in the steady proliferation of health systems solutions to non-healthcare problems. There remains a need for collaborative and committed engagement between health and other government sectors and stakeholders (e.g. transport, community safety, etc.) with the aim of jointly meeting these challenges in creative and innovative ways.

In this issue of $S A M J$, Newton et al..$^{[3]}$ explore the appropriateness of emergency medical service (EMS) responses in the eThekwini district of KwaZulu-Natal Province, SA, and suggest that current practices result ' in the wastage of expensive and limited resources that could be better utilised elsewhere. This is the challenge we face in SA emergency care: overburdened prehospital and in-hospital systems, and patients caught in the middle, shunted from one area to another. As Wallis ${ }^{[4]}$ put it in an SAMJ editorial in 2011, 'where you live determines your chance of timely, appropriate, high-quality trauma care. For patients in under-resourced areas, being able to make the distinction between health problems appropriate for a primary care clinic v. appropriate for a hospital is often impossible, and when accessing a local clinic on foot or by public transport is difficult, calling an ambulance may be a reasonable option.

According to the SA Constitution (section 27(3)), no one may be refused emergency medical care, and by implication, all citizens have the right to access emergency care. ${ }^{[5]}$ The distinctions around what is emergency care, who should provide it and where it should be provided are often out of the realm of the average person. While we do not deny that there is a need for community education and advocacy regarding the use of healthcare resources, we need to create a responsible citizenry who understand and accept the challenges of providing a critical emergency service under conditions of austerity. This will not be easy to achieve, but concerted efforts are needed to create and sustain dialogue between the public and EMS role players, a dialogue aimed at fostering meaningful and effective partnerships within the community, in order to create responsible and accountable service users but also facilitating safe 'spaces' for practitioners to render emergency care without fear of hostility or abuse. These partnerships may include community basic first-responder education and the establishment of community response networks, both of which have proven effective in empowering communities and creating engagement with emergency services. ${ }^{[6,7]}$

However, we cannot completely defer responsibility to the citizenry - the onus is on the health system and its agents at key points (EMS dispatcher, prehospital provider, clinic and hospital receiving staff) to ensure that resource-appropriate decisions are made and guided by local policies.

This is where a functional EMS system able to make these critical decisions becomes key. This is a challenge for us in SA, where far too many services are still reliant on paper-based systems. The poor and often flawed adoption of technology coupled with the absence of industry benchmarks or best practice is compounded by inappropriately trained agents and supervision. ${ }^{[8]}$ These deficiencies are evident in the failure to establish a single national emergency number and the failure to establish GIS (Geographic Information System) location of emergency incidents. Geographical modelling for the distribution and deployment of EMS resources has been proven to be a useful tool to reduce morbidity and mortality globally. ${ }^{[9,10]}$

EMS communication centres have the potential to become health intelligence resource centres, able to generate powerful data and analysis on several aspects of the health service. If we are to fully harness the potential that exists within our emergency care system, we will need to invest in our human capital - creating skilled staff able to develop and interpret these data. The SA EMS industry is not short of talent, but must become more integrated with the overall healthcare system, participatory and collaborative in terms of planning and pathways.

Our emergency services, both in and out of hospital, are overburdened. Creating equitable access to care for all, rural and urban, is an ongoing challenge and needs a number of components: an informed and engaged community, a skilled and empowered EMS communication system, and an integrated healthcare system with policies and protocols to guide patients to the most appropriate site to receive appropriate care. And in the midst of all the resource protection, we must remember that there is still a patient - a person at the centre of it all.

\section{Shaheem de Vries}

Director, Western Cape Emergency Medical Services, Cape Town, South Africa

\section{Heike Geduld}

Western Cape Emergency Medical Services, Cape Town, South Africa, and Division of Emergency Medicine, Faculty of Health Sciences, University of Cape Town

Corresponding author: H I Geduld (heike.geduld@uct.ac.za)

1. Harris B, Goudge J, Ataguba JE, et al. Inequalities in access to health care in South Africa. J Public Health Policy 2011;32(Suppl 1):S102-S123. [http://dx.doi.org/10.1057/jphp.2011.35]

. McLaren Z, Ardington C, Leibbrandt M. Distance as a Barrier to Health Care Access in South Africa A Southern Africa Labour and Development Research Unit Working Paper Number 97. Cape Town: SALDRU, University of Cape Town, 2013.

3. Newton PR, Naidoo R, Brysiewicz P. The appropriateness of emergency medical services (EMS) responses in the eThekwini district of KwaZulu-Natal, South Africa. S Afr Med J 2015;105(10):844847. [http://dx.doi.org/10.7196/SAMJnew.8273]

4. Wallis LA. Trauma care in South Africa - a call to arms. S Afr Med J 2011;101(3):171.

5. South Africa. The Constitution of the Republic of South Africa. 1996. www.justice.gov.za (accessed 3 September 2015).

6. Sun J, Wallis LA. The emergency first aid responder system model: Using community members to assist life-threatening emergencies in violent, developing areas of need. Emerg Med J 2012;29(8):673678. [http://dx.doi.org/10.1136/emermed-2011-200271]

7. Sun J, Shing R, Twomey M, Wallis LA. A strategy to implement and support pre-hospital emergency Sun J, Shing R, Twomey M, Wallis LA. A strategy to implement and support pre-hospital emergency
medical systems in developing, resource-constrained areas of South Africa. Injury 2014;45(1):31-38. medical systems in developing, resource-constra

[http://dx.doi.org/10.1016/j.injury.2012.08.015]
8. Mould-Millman N, de Vries S, Stein C, et al. Developing emergency medical dispatch systems in Africa - recommendations of the African Federation for Emergency Medicine/International Academies of Emergency Dispatch Working Group. African Journal of Emergency Medicine 2015;5(3):141-147. [http://dx.doi.org/10.1016/j.afjem.2015.06.005]

9. Vanderschuren M, McKune D. Emergency care facility access in rural areas within the golden hour: Western Cape case study. Int J Health Geogr 2015;14(1):5-13. [http://dx.doi.org/10.1186/1476072X-14-5]

10. Kobusingye OC, Hyder AA, Hicks ER, Mock C, Joshipura M. Emergency medical systems in low-and middle-income countries: Recommendations for action. Bull World Health Organ 2005:83(8):626631. [http://dx.doi.org/10.1590/S0042-96862005000800017]

S Afr Med J 2015;105(10):816. DOI:10.7196/SAMJ.9980 